\title{
Seeking Completeness for high- $z$ ULIRGs: from SCUBA to Herschel
}

\author{
Caitlin M. Casey ${ }^{1} \dagger$ \\ ${ }^{1}$ Institute for Astronomy \\ University of Hawai'i \\ 2680 Woodlawn Dr \\ Honolulu, HI 96822, USA \\ email: cmcasey@ifa.hawaii.edu
}

\begin{abstract}
Selection effects in the submillimeter/far-infrared inhibit detailed analysis of extreme starbursts in the early Universe. Submillimeter Galaxy (SMG) detection and characterization biases against warm temperature SEDs $T_{\text {dust }} \sim 50 \mathrm{~K}$, against the highest redshift ULIRGs $z>3$, and has so far been limited to a small sample of $\sim 100$ sources that have reliably identified optical or radio counterparts and spectroscopic redshifts. Recent observations by Herschel PACS and SPIRE in the $\sim 70-500 \mu \mathrm{m}$ wavelength range provide a more complete census of high- $z$ ULIRG activity than previous SMG surveys. However, source confusion and lack of spectroscopic identification of reliable counterparts limit the analysis of extreme ULIRGs to the most basic calculations, frought with a priori assumptions (e.g. $T_{d u s t} /(1+z)$ degeneracy, far-IR/radio relation, confusion boosting factors, etc). Herein I address the biases on conclusions of SMG work and I demonstrate the need for thorough, spectroscopic follow-up of high- $z$ ULIRGs in order to infer their physical conditions.
\end{abstract}

Keywords. galaxies-starburst, galaxies-infrared

\section{Introduction}

Ultraluminous infrared galaxies (ULIRGs) exhibit the most extreme star formation rates in the Universe. At early epochs $(z>1)$, ULIRG activity contributes significantly to the build-up of stellar mass through intense star-forming bursts (with $\tau<100 \mathrm{Myr}$ and $\mathrm{SFR}>500 \mathrm{M}_{\odot} \mathrm{yr}^{-1}$ ). Since the observed properties of these starbursts are short-lived and extreme, they are thought to be triggered by the collision of gas-rich disk galaxies and serve as a fundamental transition phase to luminous active galactic nuclei (Sanders et al. 1988a,b). While ULIRGs are likely responsible for the formation of massive elliptical galaxies in the local Universe, much about the population is still unknown due to limitations in far-infrared (FIR) observations, strong selection biases, sample inhomogeneity and spectroscopic incompleteness.

Submillimeter Galaxies (SMGs, a subset of $z>1$ ULIRGs; Smail et al., 2002) put powerful constraints on galaxy evolution theories and the environments of heavy star formation, but their selection at $850 \mu \mathrm{m}$ is susceptible to strong temperature biasing (Blain et al., 2004; Chapman et al., 2004; Casey et al., 2009b). In addition, spectroscopic followup of SMGs is reliant upon radio counterpart identification, which tends to exclude very high redshift sources $(z>3)$ due to the fall off in radio luminosity. This implies that a significant fraction of high- $z$ ULIRGs have yet to be discovered and characterized.

The current generation of infrared (IR) and radio observatories, including the Herschel Space Observatory, the Extended Very Large Array (EVLA), SCUBA-2, and the

$\dagger$ Hubble Fellow 
Atacama Large Millimeter Array (ALMA), will dramatically improve our census of luminous starbursts in the early Universe and allow detailed study of their formation and evolution. Herschel has begun working towards completeness for high- $z$ ULIRGs by selecting several hundred 70-160 $\mu \mathrm{m}$ (PACS) and 250-500 $\mu \mathrm{m}$ (SPIRE) luminous sources, however, poor multi-wavelength counterpart identification with uncertain FIR positions and a lack of spectroscopic measurements renders detailed, physical analysis very difficult on a source-by-source basis.

In these proceedings, I first revisit the work on SMGs from the past decade and their biases. Then I use this summary of SMG work to infer what types of analysis will be necessary for yet-uncharacterized Herschel sources, particularly emphasizing the necessity of extensive spectroscopic follow-up observations using both rest-frame UV/optical and millimeter-line detection techniques.

\section{Conclusions from SMGs}

The first SMGs were first selected at $850 \mu \mathrm{m}$ using the Submillimeter Common User Bolometric Array (SCUBA) on the JCMT on Mauna Kea, Hawaii (Hughes et al. 1998; Barger et al. 1998, 1999, 2000, Cowie et al. 2002). Since there is a negative k-correction on the Rayleigh-Jeans tail of dust-generated black body spectra, it was quickly inferred that $850 \mu \mathrm{m}$ flux counts were dominated by sources at $2<z<4$, however the large beamsize of observations in the IR ( 15-20" with SCUBA) made the sources' multiwavelength follow-up challenging. Below we describe the most striking discoveries on SMGs in the past decade.

SMG Selection

Chapman et al. 2005 present 75 spectroscopic redshifts for SMG radio-identified counterparts, the most extensive redshift survey of SMGs to date. The SMGs' radio counterparts (chosen based on the FIR/radio correlation, see below) were then surveyed for prominent emission lines (Lyman- $\alpha$, [OII], CIV, H- $\beta$ ) using the Keck instruments LRIS and DEIMOS (3000-10000A). A mean redshift of $\langle z\rangle \approx 2.2$ and mean $\langle S F R\rangle \approx 900 \mathrm{M}_{\odot} \mathrm{yr}^{-1}$ were inferred (assuming that FIR luminosity scales with SFR as in Kennicutt et al. 1998). However, this spectroscopic survey suffered from selection biases which excluded several populations of high- $z$ starbursts:

- SMGs without radio counterparts (thought to lie at the highest redshifts $z>3$ thus below the detection limit at $1.4 \mathrm{GHz})$,

- SMGs too optically obscured to detect prominent emission lines,

- ULIRGs not initially detected at $850 \mu \mathrm{m}$ due to their slightly warmer dust temperatures than SMGs (noting that flux density has a strong temperature dependence, e.g. $S_{850} \propto T_{d u s t}^{-3.5} L_{F I R}$ at $z \sim 2$, see Blain et al. 2004, Casey et al. 2009b and Figure 1), and

- Fainter starbursts with $S_{850} \sim 1-5 \mathrm{mJy}$, too confused to distinguish individually, but whose emission is thought to dominate the submillimeter background (see Cowie et al. 2002, Wang et al. 2011).

\section{Goals for Herschel Samples}

Constraining the FIR emission from high- $z$ ULIRGs is paramount to understanding star formation in the early Universe. SMGs have accounted for only some of the discrepancy between predictions of the cosmic star formation rate density (SFRD) from UV/optical surveys and the Cosmic Infrared Background (CIB; Dwek et al., 1998). Even the most current work on the cosmic SFRD (e.g. Bouwens et al., 2009) has difficulty accounting for the ULIRG contribution at $z>2$ since it relies on biased surface density 
estimates from SCUBA and MAMBO $(850 \mu \mathrm{m}, 1.2 \mathrm{~mm})$. While Herschel has begun to provide near complete number counts on FIR bright sources, the limitation in spectroscopic samples prevents detailed analysis of dust SED type, molecular gas and dynamical structure. Also fundamental to the work on high-redshift galaxies is the calibration of star formation rate indicators, from the rest-UV, to $24 \mu \mathrm{m}$, PAH, FIR, or radio, all of which are presently subject to large assumptions due to a lack of data or accurate redshifts.

The evolutionary history of SMGs as analyzed in the previous session must be tested against a complete sample of high- $z$ ULIRGs in order to untangle the dependence of SMG analysis on spectroscopic or selection biases. Spectroscopic redshifts are needed to lift the degeneracy between dust temperature and redshift uncertainty, to follow up sources in $\mathrm{CO}$ (which is necessary for $\sim 10^{12}$ sources before ALMA is in full array science mode), and to lift the dependence on assumptions like the FIR/radio correlation or dust SED type. The method of identifying a source's redshift through a CO detection in the FIR is promising (used currently with the Z-SPEC instrument at CSO and APEX; Bradford et al. 2009), however, it requires a significant time investment per source and is so far limited to the brightest subset of objects $\left(>10^{13} \mathrm{~L}_{\odot}\right.$ or lensed); the full array mode for ALMA will improve upon this method's efficiency and push towards lower luminosities, however this will be of limited or incomplete access for the next several years. Therefore, traditional spectroscopic followup in the rest-frame ultraviolet and optical is the most efficient way to spectroscopically confirm large samples of 250-500 $\mu \mathrm{m}$ Herschel sources, despite the sample biasing discussed in the previous section.

The eventual goal in this field is to build a sizable sample of diverse, spectroscopically confirmed ULIRGs so that the extreme starburst luminosity function and the ULIRG

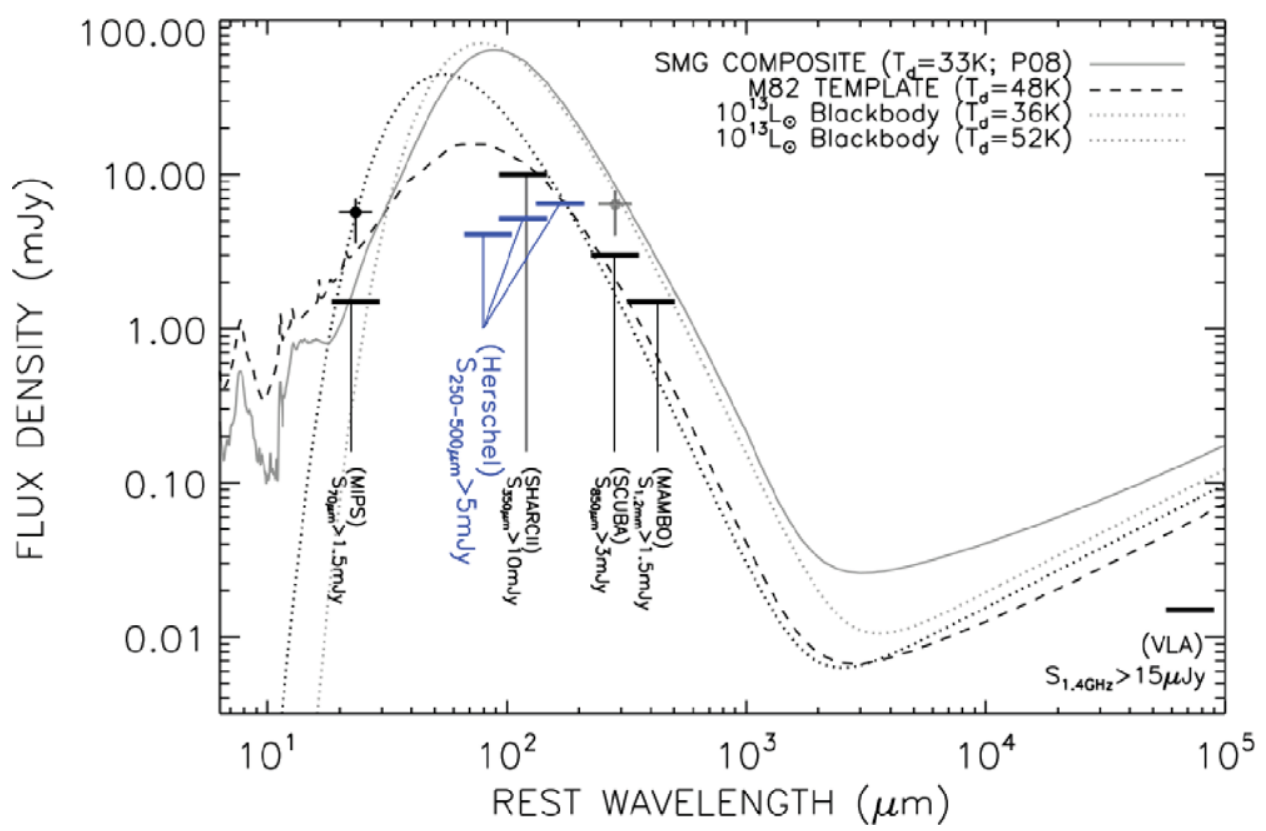

Figure 1. Typical FIR SEDs of local and high-z ULIRGs. SMGs (gray composite) have $\mathrm{T}_{\text {dust }}=36 \mathrm{~K}$, much colder than the local ULIRG M82 (dashed line). Hot-dust and cold-dust ULIRG blackbodies (dotted lines) contrast significantly in their flux densities at $70 \mu \mathrm{m}$ and $850 \mu \mathrm{m}$ and all intermediate wavelengths. Bars mark the typical depth of FIR blank field surveys, and the improvement which Herschel brings as a FIR probe is highlighted by the blue detection limit bars. 
contribution to the cosmic star formation rate density (SFRD) will be constrained. An accurate estimate of the extreme starburst luminosity function, if broken into $\Delta z \sim 0.5$ redshift bins and requires spectroscopic redshifts for $>500$ sources to avoid being limited by poisson statistics (assuming a coarse $\log$ (luminosity) binning of $\sim 0.5$ dex). Providing a more conservative delineation of the luminosity function, with only 3 redshift bins (e.g. $\sim 1.0-1.7,1.7-2.3,>2.3)$ would lower the required target numbers slightly $(\sim 300$ 400 sources) except it would make it impossible to separate sub-populations for further analysis. For example, separating the luminosity function into hot-dust and cold-dust dominated components might shed light on the physical drivers of their physical properties. Similarly, if we wish to precisely measure a turnover in the slope of the luminosity function, large samples (>500) of spectroscopically confirmed ULIRG redshifts are desperately needed. Efforts to measure the high-redshift ULIRG luminosity function from the currently limited $\sim 100$ object sample (which consists of $\sim 75$ SMGs with published redshifts and $\sim 25$ ULIRGs selected using other methods) have failed on the basis of poor statistics (e.g. Pope et al. 2006). In turn, the luminosity function will give an accurate estimate to volume density, shedding light on the ULIRG contribution to the star formation history of the Universe.

\section{References}

Barger A. J., Cowie L. L., \& Richards E. A., 2000, AJ, 119, 2092

Barger A. J., Cowie L. L., Sanders D. B., Fulton E., Taniguchi Y., Sato Y., Kawara K., \& Okuda H., 1998, Nature, 394, 248

Barger A. J., Cowie L. L., Smail I., Ivison R. J., Blain A. W., \& Kneib J., 1999, AJ, 117, 2656

Blain A. W., Chapman S. C., Smail I., \& Ivison R., 2004, ApJ, 611, 725

Bouwens R. J., Illingworth G. D., Franx M., Chary R., Meurer G. R., Conselice C. J., Ford H., Giavalisco M., \& van Dokkum P., 2009, ApJ, 705, 936

Bradford C. M., Aguirre J. E., Aikin R., Bock J. J., Earle L., Glenn J., Inami H., Maloney P. R., Matsuhara H., Naylor B. J., Nguyen H. T., \& Zmuidzinas J., 2009, ApJ, 705, 112

Casey C. M., et al., 2009, MNRAS, 399, 121

Chapman S. C., Blain A. W., Smail I., \& Ivison R. J., 2005, ApJ, 622, 772

Chapman S. C., Smail I., Blain A. W., \& Ivison R. J., 2004, ApJ, 614, 671

Cowie L. L., Barger A. J., \& Kneib J.,

Dwek E., Arendt R. G., Hauser M. G., Fixsen D., Kelsall T., Leisawitz D., Pei Y. C., Wright E. L., Mather J. C., Moseley S. H., Odegard N., Shafer R., Silverberg R. F., \& Weiland J. L., 1998, ApJ, 508, 106

Engel H., et al., 2010, ApJ, 724, 233

Hughes D. H., Serjeant S., Dunlop J., Rowan-Robinson M., Blain A., Mann R. G., Ivison R., Peacock J., Efstathiou A., Gear W., Oliver S., Lawrence A., Longair M., Goldschmidt P., \& Jenness T., 1998, Nature, 394, 241

Kennicutt Jr. R. C., 1998, ApJ, 498, 541

Pope A., Scott D., Dickinson M., Chary R., Morrison G., Borys C., Sajina A., Alexander D. M., Daddi E., Frayer D., MacDonald E., \& Stern D., 2006, MNRAS, 370, 1185

Sanders D. B., Soifer B. T., Elias J. H., Madore B. F., Matthews K., Neugebauer G., \& Scoville N. Z., 1988, ApJ, 325, 74

Sanders D. B., Soifer B. T., Elias J. H., Neugebauer G., \& Matthews K., 1988, ApJL, 328, L35

Smail I., Ivison R. J., Blain A. W., \& Kneib J., 2002, MNRAS, 331, 495

Wang W., Cowie L. L., Barger A. J., \& Williams J. P., 2011, ApJL, 726, L18+ 